\title{
Deconvolution Filtering for Nonlinear Stochastic Systems with Randomly Occurring Sensor Delays via Probability-Dependent Method
}

\author{
Yuqiang Luo, ${ }^{1,2}$ Guoliang Wei, ${ }^{1}$ Hamid Reza Karimi, ${ }^{3}$ and Licheng Wang ${ }^{1}$ \\ ${ }^{1}$ Shanghai Key Lab of Modern Optical System, Department of Control Science and Engineering, University of Shanghai for Science and \\ Technology, Shanghai 200093, China \\ ${ }^{2}$ Informatization Office, University of Shanghai for Science and Technology, Shanghai 200093, China \\ ${ }^{3}$ Department of Engineering, Faculty of Engineering and Science, University of Agder, N-4898 Grimstad, Norway
}

Correspondence should be addressed to Guoliang Wei; guoliang.wei1973@gmail.com

Received 12 April 2013; Accepted 20 May 2013

Academic Editor: Zidong Wang

Copyright (C) 2013 Yuqiang Luo et al. This is an open access article distributed under the Creative Commons Attribution License, which permits unrestricted use, distribution, and reproduction in any medium, provided the original work is properly cited.

\begin{abstract}
This paper deals with a robust $H_{\infty}$ deconvolution filtering problem for discrete-time nonlinear stochastic systems with randomly occurring sensor delays. The delayed measurements are assumed to occur in a random way characterized by a random variable sequence following the Bernoulli distribution with time-varying probability. The purpose is to design an $H_{\infty}$ deconvolution filter such that, for all the admissible randomly occurring sensor delays, nonlinear disturbances, and external noises, the input signal distorted by the transmission channel could be recovered to a specified extent. By utilizing the constructed Lyapunov functional relying on the time-varying probability parameters, the desired sufficient criteria are derived. The proposed $H_{\infty}$ deconvolution filter parameters include not only the fixed gains obtained by solving a convex optimization problem but also the online measurable timevarying probability. When the time-varying sensor delays occur randomly with a time-varying probability sequence, the proposed gain-scheduled filtering algorithm is very effective. The obtained design algorithm is finally verified in the light of simulation examples.
\end{abstract}

\section{Introduction}

Filtering technology is extensively used in many domains, and an important task of filtering is to effectively restore the original signal by removing the distortion from the received signal. The deconvolution method is very useful in filtering field and has been made use of by some kinds of filters, such as self-tuning deconvolution filter and Wiener inverse filter. However, the design process of the deconvolution filter is always difficult because the delivery channels are frequently corrupted by some disturbances, such as nonlinear disturbances, external noises, and sensor delays. Therefore it is very interesting and important to solve the problem encountered in the design procedure of deconvolution filters. In the past decades, considerable attention has been paid to the analysis and design of many kinds of deconvolution filters, and some results have been published. For more details, we refer the readers to $[1-3]$ and the references therein.

As is well known, in some practical fields, such as engineering, biological, medical, and economic systems, and health community, the sensor data is occasionally delayed before they arrive at their respective destinations [4-6]. The occurrence of sensor delay may mainly be caused by the limitations, such as the limited bandwidth of the communication channel, intermittent sensor failure in the measurement, random network congestion, and accidental loss of some collected data in a very noisy environment [6-9]. However, the majority of deconvolution filtering algorithms are based on the measurement outputs without delays. In such case, the traditional filter may fail to work. Hence, in the past decades the filtering problem for the systems with sensor delays has been attracting considerable research interests; see, 
for example, $[5,7,10-12]$. Owing to the uncertainty that is widespread in the field of practical engineering, stochastic model has gained more and more attention in many fields, such as physics, economic systems, geomorphology, and gene regulatory networks, and a large number of literatures have been published; see, for example, [4, 13-18].

It should be pointed out that, so far, the Bernoulli distribution has been employed to model some randomly occurring phenomena, such as randomly occurring missing measurements [10] and randomly occurring saturation [7]. This model with time-invariant probability has become an effective model of sensor delays. Nevertheless, in the engineering environment, such as industrial automation, unmanned vehicles, real-time distributed decision-making and multiplexed data communication networks [5], and an asynchronous time-division-multiplexed network [6], the sensor delays often occur in a random way and satisfy a time-varying probability distribution. At the same time, the classical filters with fixed gains cannot adapt to the actual cases. Therefore, there is an urgent need to develop new filtering approaches for the systems with randomly occurring sensor delays (ROSDs), and some efforts have been made in this regard so far; see, for example, $[6,7]$. And yet, up to now, to the best of authors' knowledge, the gain-scheduled $H_{\infty}$ deconvolution filtering is still open for discrete-time stochastic systems with randomly occurring sensor delays. It is, therefore, in this paper, we aim to develop an effective gain-scheduled deconvolution filtering algorithm for the discrete-time stochastic systems with ROSDs, which is of both theoretical importance and practical significance.

The main contribution of this paper is mainly triplex: (1) for the randomly occurring sensor delay which is one kind of the information incomplete, we exploit a stochastic variable sequence satisfying time-varying Bernoulli distributions to represent the situation of the delayed measurement; (2) a time-varying Lyapunov functional dependent on the distribution probability has been developed and applied to improve the performance of the $H_{\infty}$ deconvolution filters; (3) a new filtering problem with a gainscheduling approach is addressed for a class of discrete-time nonlinear stochastic systems with randomly occurring sensor delays. It is worth mentioning that, since the considered system involves the probabilistic sensor delays, the sectorlike bounded nonlinearity, and the multiplicative noises, it is comprehensive and reasonable. Thanks to the proposed time-varying $H_{\infty}$ deconvolution filter which is designed by employing the gain-scheduling technique, the proposed filtering algorithm can exactly estimate the original input. On account of this merit, the proposed design scheme is more effective and practical.

The rest of this paper is organized as follows. In Section 2, we construct a gain-scheduled deconvolution filter for a class of discrete-time stochastic systems with randomly occurring sensor delays, in which the desired filter gains contain two parts, the fixed gain and time-varying one which is dependent upon the time-varying probability. In Section 3, a sufficient condition is derived to guarantee the exponential stability of the augmented system, and the proposed filter is given. By means of constructing Lyapunov functional, we make an

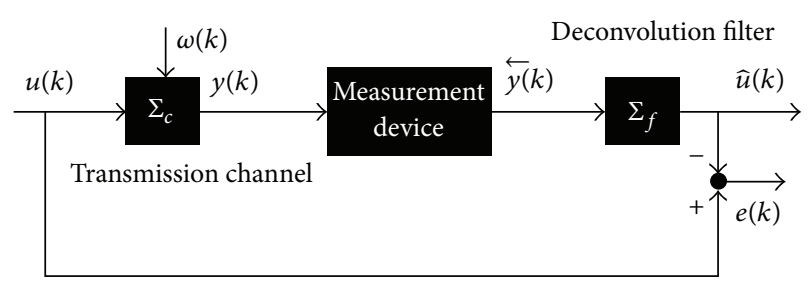

FIgURE 1: The deconvolution filtering system.

assay of the stability for the augmented systems. With the help of the proposed probability-dependent Lyapunov functional, we derived simultaneously another condition indicating the robust ability of the deconvolution filter. A mathematical technique is used to transform the infinite number of inequalities into a finite form. The filter gains are derived from a gain-scheduling approach by resorting to solve a convex optimization problem. In Section 4, an numerical example is presented to demonstrate the reasonable structure and high reliability of the proposed filter. The last section, Section 5, sums up all the arguments in this paper.

Notation. In this paper, $\mathbb{R}^{n}, \mathbb{R}^{n \times m}$, and $\mathbb{\square}^{+}$denote, respectively, the $n$-dimensional Euclidean space and the set of all $n \times m$ real matrices, the set of all positive integers. $|\cdot|$ refers to the Euclidean norm in $\mathbb{R}^{n}$. I denotes the identity matrix of compatible dimension. The notation $X \geq Y$ (resp., $X>Y$ ), where $X$ and $Y$ are symmetric matrices, means that $X-Y$ is positive semidefinite (resp., positive definite). For a matrix $M, M^{T}$ and $M^{-1}$ represent its transpose and inverse, respectively. The shorthand $\operatorname{diag}\left\{M_{1}, M_{2}, \ldots, M_{n}\right\}$ denotes a block diagonal matrix with diagonal blocks being the matrices $M_{1}, M_{2}, \ldots, M_{n}$. In symmetric block matrices, the symbol $*$ is used as an ellipsis for terms induced by symmetry. Matrices, if they are not explicitly stated, are assumed to have compatible dimensions.

\section{Problem Formulation}

In this paper, the considered stochastic deconvolution filtering system structure is shown in Figure 1. In the system, the input signal $u(t)$ is transmitted through the channel $\Sigma_{c}$ :

$$
\begin{aligned}
&\left(\Sigma_{c}\right): x_{c}(k+1)= A_{c} x_{c}(k) \\
&+B_{c} u(k)+N_{c} f(z(k)) \\
&+M_{c} x_{c}(k) \omega(k), \\
& y(k)=C_{c} x_{c}(k), \quad x_{c}(0)=x_{0},
\end{aligned}
$$

where $x_{c}(k) \in \mathbb{R}^{n}$ is the state, $u(k) \in L_{2}[0, \infty)$ is the exogenous input signal, $y(k)$ is the actual output, and $x_{0}$ is the initial state. $\omega(k)$ is a one-dimensional Gaussian white noise sequence satisfying $\mathbb{E}\{\omega(k)\}=0$ and $\mathbb{E}\left\{\omega^{2}(k)\right\}=\sigma^{2}$, and $z(k)=Z x_{c}(k)$. For the convenience, $u(k), y(k)$, and $\omega(k)$ are all assumed as scalars. $A_{c}, B_{c}, C_{c}, M_{c}, N_{c}$, and $Z$ are constant matrices with appropriate dimensions. 
Remark 1. The deconvolution filtering problem involves the estimation of the signal inputted to a communication channel where the output measurements are disturbed by the channel noise. The channel can be represented by a dynamical system, such as ship roll stabilization systems. In the ideal condition, the input signal would be sent perfectly through the transmission channel without any external influences; however, in the real-world case, from the point of engineering application, most of the communication channels are of limited capacity and suffered from some uncertainties in transmission process such as large environmental noise, channel congestion, and intermittent changes of the signal intensity, and therefore, a few issues have inevitably emerged, for example, channelinduced time delay, the nonlinearity disturbances, channel fading, and so on. In order to describe the channel model $\sum_{c}$ closer in nature, nonlinearity disturbances and stochastic noises are both taken into consideration in the channel model (1) in this paper.

The vector-valued nonlinear disturbance $f(\cdot)$ satisfies the following sector-bounded condition with $f(0)=0$ :

$$
\left[f(z(k))-F_{1} z(k)\right]^{T}\left[f(z(k))-F_{2} z(k)\right] \leq 0,
$$

where $F_{1}$ and $F_{2}$ are constant real matrices of appropriate dimensions and $F=F_{2}-F_{1}$ is a symmetric positive definite matrix. It is customary that such nonlinear function $f(\cdot)$ is called to belong to the sector $\left[\begin{array}{ll}F_{1} & F_{2}\end{array}\right]$. In this case, the nonlinear function $f(z(k))$ can be decomposed into a linear part and a nonlinear part as

$$
f(z(k))=F_{1} z(k)+f_{s}(z(k)),
$$

and it is easy to follow from (3) that

$$
f_{s}^{T}(z(k))\left(f_{s}(z(k))-F z(k)\right) \leq 0 .
$$

The measurement outputs $\overleftarrow{y(k)}$ with sensor delays are described by

$$
\overleftarrow{y(k)}=(1-\omega(k)) y(k)+\omega(k) y(k-d)
$$

where $d \in \mathbb{Q}^{+}$is the sensor delay and $\omega(k) \in \mathbb{R}$ is a random white sequence characterizing the probabilistic sensor delays and obeys the following Bernoulli distribution with timevarying probability:

$$
\begin{gathered}
\operatorname{Prob}\{\omega(k)=1\}=\mathbb{E}\{\omega(k)\}=p(k), \\
\operatorname{Prob}\{\omega(k)=0\} \\
=1-\mathbb{E}\{\omega(k)\}=1-p(k),
\end{gathered}
$$

where $p(k)$ is a time-varying positive scalar sequence that belongs to $\left[p_{1} p_{2}\right] \subseteq\left[\begin{array}{ll}0 & 1\end{array}\right]$ with the constants $p_{1}$ and $p_{2}$ being the lower and upper bounds of $p(k)$.

Remark 2. The sensor delays may randomly occur due to some environment reasons, and a Bernoulli distribution model has been introduced in $[6,7]$ to describe such random phenomenon. However, the Bernoulli distributions in this literature are assumed to be time invariant, which is apparently conservative to deal with the time-varying cases of randomly occurred sensor delays for time-varying systems. In this paper, we will utilize a stochastic variable sequence in (7) satisfying time-varying Bernoulli distributions to express the randomly intermittent phenomenon of the discussed sensor delays.

In order to recover the source signal $u(k)$, the following deconvolution filter structure is considered in this paper:

$$
\begin{gathered}
\Sigma_{f}: x_{f}(k+1)=A_{f} x_{f}(k)+B_{f} \overleftarrow{y(k)} \\
\widehat{u}(k)=C_{f} x_{f}(k)+D_{f} \overleftarrow{y(k)}
\end{gathered}
$$

where $x_{f}(k) \in \mathbb{R}^{n}$ is the filter state and the matrices $A_{f}, B_{f}$, $C_{f}$, and $D_{f}$ are filter parameters to be determined and have the following forms:

$$
\begin{array}{ll}
A_{f}=A_{f 0}+p(k) A_{f p}, & B_{f}=B_{f 0}+p(k) B_{f p}, \\
C_{f}=C_{f 0}+p(k) C_{f p}, & D_{f}=D_{f 0}+p(k) D_{f p},
\end{array}
$$

where $A_{f 0}, A_{f p}, B_{f 0}, B_{f p}, C_{f 0}, C_{f p}, D_{f 0}$, and $D_{f p}$ are the constant filter gains to be designed and $p(k)$ is the time-varying probability that can be estimated/measured via statistical tests in real time.

Remark 3. Deconvolution filter is a restoration algorithm to remove a wavelet by utilizing a reverse process of convolution. Comparing with conventional filters, it not only can estimate a signal embedded in noise but also can remove the effect of any distortion in the channel systems. Furthermore, it can deal with unknown boundary problem and spatially varying blurs. It is worth mentioning that, different from many conventional deconvolution filters with only constant parameters, the proposed filter gains in (10) include two kinds of filter gains: the fixed parameters $A_{f 0}, A_{f p}, B_{f 0}, B_{f p}, C_{f 0}$, $C_{f p}, D_{f 0}$, and $D_{f p}$ and the time-varying parameter $p(k)$. The designed filter can be scheduled with the time-varying probability, which is able to adapt to changing circumstances naturally. It can be divided into the following several steps. Firstly, compute the constant gains $A_{f 0}, A_{f p}, B_{f 0}, B_{f p}, C_{f 0}$, $C_{f p}, D_{f 0}$, and $D_{f p}$ in terms of the main results that will be developed in this paper. Secondly, estimate/measure the time-varying probability $p(k)$ by statistical tests in real time. Lastly, the filter gains can be derived from (10). Obviously, the gain-scheduled filter is reasonable and the conservatism of which can be reduced since more information about the sensor delay phenomenon is utilized. Note that gainscheduled technique filtering and control problems have become a hot topic and have been intensively researched in the past decades; see, for example, [19-23]. 
By setting $\xi(k)=\left[\begin{array}{ll}x_{c}^{T}(k) & x_{f}^{T}(k)\end{array}\right]^{T}$ and the signal error as $e(k)=u(k)-\widehat{u}(k)$, the dynamics of the filtering process can be derived from (1)-(6) and (8)-(9) as follows:

where

$$
\begin{gathered}
A(p(k))=\left[\begin{array}{cc}
A_{c} & 0 \\
(1-p(k)) B_{f} C_{c} & A_{f}
\end{array}\right], \\
B=\left[\begin{array}{c}
B_{c} \\
0
\end{array}\right], \quad M=\left[\begin{array}{c}
M_{c} \\
0
\end{array}\right], \\
D(p(k))=\left[\begin{array}{cc}
0 & 0 \\
p(k) B_{f} C_{c} & 0
\end{array}\right], \\
K(p(k))=\left[\begin{array}{c}
0 \\
B_{f} C_{c}
\end{array}\right], \quad N=\left[\begin{array}{c}
N_{c} \\
0
\end{array}\right], \\
E_{c d f}(p(k))=\left[\begin{array}{ll}
(1-p(k)) D_{f} C_{c} & C_{f}
\end{array}\right], \\
E_{d f}(p(k))=\left[\begin{array}{ll}
D_{f} C_{c} & 0
\end{array}\right], \quad J=\left[\begin{array}{ll}
I & 0
\end{array}\right] .
\end{gathered}
$$

Definition 4. The augmented filtering system (11) is said to be exponentially mean-square stable if, with $u(k) \equiv 0$, there exist

$$
\mathbb{E}\left\{\|\xi(k)\|^{2}\right\} \leq \alpha \tau^{k} \sup _{-d \leq i \leq 0} \mathbb{E}\left\{\|\xi(i)\|^{2}\right\}, \quad k \in \mathbb{\mathbb { Q }}^{+} .
$$

$$
\begin{aligned}
\xi(k+1)= & A(p(k)) \xi(k) \\
& +D(p(k)) \xi(k-d)+B u(k) \\
& +N f(z(k))+M J \xi(k) \omega(k) \\
& +(p(k)-\omega(k)) K(p(k)) J \xi(k) \\
& +(\varrho(k)-p(k)) K(p(k)) J \xi(k-d), \\
e(k)= & -\left[E_{c d f}(p(k))+(p(k)-\varrho(k)) E_{d f}(p(k))\right] \xi(k) \\
& -\left[p(k) E_{d f}(p(k))+(\varrho(k)-p(k)) E_{d f}(p(k))\right] \\
& \times \xi(k-d)+u(k),
\end{aligned}
$$
constant $\alpha>0$ and $\tau \in(0,1)$ such that

Definition 5. Given a scalar $\gamma>0$, the dynamics of the augmented systems (11)-(12) are said to be stochastically stable with disturbance attenuation level $\gamma$ if it is exponentially mean-square stable, and under zero initial condition, $\|e(k)\|_{\mathbb{E}_{12}}<\gamma\|u(k)\|_{l 2}$ holds for all nonzero $u(k) \in l_{2}[0, \infty)$, where

$$
\|e(k)\|_{\mathbb{E}_{l 2}}:=\left(\mathbb{E}\left\{\sum_{k=1}^{\infty}|e(k)|^{2}\right\}\right)^{1 / 2} .
$$

This paper aims to design a $H_{\infty}$ deconvolution filter to recover a input signal $u(k)$ transmitted through a noised channel (1)-(2) such that the closed-loop systems (11)-(12) are stochastically stable with disturbance attenuation level $\gamma$.

\section{Main Results}

In the proof procedure of theorems presented in this paper, we will use the following lemma.

Lemma 6 ((Schur complement), see [24]). Given constant matrices $\Sigma_{1}, \Sigma_{2}$, and $\Sigma_{3}$ where $\Sigma_{1}=\Sigma_{1}^{T}$ and $0<\Sigma_{2}=\Sigma_{2}^{T}$, then $\Sigma_{1}-\Sigma_{3}^{T} \Sigma_{2}^{-1} \Sigma_{3} \geq 0$ if and only if

$$
\left[\begin{array}{cc}
\Sigma_{1} & \Sigma_{3}^{T} \\
\Sigma_{3} & \Sigma_{2}
\end{array}\right] \geq 0 \text { or } \quad\left[\begin{array}{cc}
\Sigma_{2} & \Sigma_{3} \\
\Sigma_{3}^{T} & \Sigma_{1}
\end{array}\right] \geq 0 \text {. }
$$

In the following theorem, a sufficient condition is derived for the augmented filtering dynamics (11)-(12) with $u(k) \equiv$ 0 to ensure the exponential mean-square stability of the considered dynamics.

Theorem 7. Consider the augmented filtering system (11) with $u(k) \equiv 0$. If there exist positive-definite matrix $Q_{d}>0$, $Q(p(k))>0$, and matrix $S$ such that the following matrix inequalities

$$
\left[\begin{array}{cccccc}
Q_{d}-Q(p(k)) & * & * & * & * & * \\
0 & -Q_{d} & * & * & * & * \\
F Z J & 0 & -2 I & * & * & * \\
\sigma^{2} S^{T} M J & 0 & 0 & -\sigma^{2} \widetilde{\Gamma}(k) & * & * \\
\theta(k) S^{T} K(p(k)) J & -\theta(k) S^{T} K(p(k)) J & 0 & 0 & -\theta(k) \widetilde{\Gamma}(k) & * \\
S^{T}\left(A(p(k))+N F_{1} Z J\right) & S^{T} D(p(k)) & S^{T} N & 0 & 0 & -\widetilde{\Gamma}(k)
\end{array}\right]<0
$$

hold, where

$$
\begin{gathered}
\widetilde{\Gamma}(k)=-Q(p(k+1))+S+S^{T}, \\
\theta(k)=p(k)(1-p(k)),
\end{gathered}
$$

then the augmented dynamics (11) is exponentially stable in mean square sense.
Proof. Now, we will show the exponential mean-square stability of the augmented system (11). To this end, define the Lyapunov functional as

$$
V(k):=\xi^{T}(k) Q(p(k)) \xi(k)+\sum_{s=k-d}^{k-1} \xi^{T}(s) Q_{d} \xi(s),
$$


where $Q(p(k))$ is a time-varying positive definite matrix sequence dependent on the time-varying probability $p(k)$ :

$$
\begin{aligned}
& \mathbb{E}\{\Delta V(k)\} \\
&=\mathbb{E}\left\{\xi^{T}(k+1) Q(p(k+1))\right. \\
& \quad \times \xi(k+1)-\xi^{T}(k) Q(p(k)) \xi(k) \\
&+\xi^{T}(k) Q_{d} \xi(k)-\xi^{T}(k-d) \\
&\left.\quad \times Q_{d} \xi(k-d)\right\} .
\end{aligned}
$$

Noting that $\mathbb{E}\{\omega(k)-p(k)\}=0, \mathbb{E}\left\{(\omega(k)-p(k))^{2}\right\}=p(k)(1-$ $p(k)), \mathbb{E}\{\omega(k)\}=0$, and $\mathbb{E}\left\{\omega^{2}(k)\right\}=\sigma^{2}$, it can be obtained from (11) that

$$
\begin{aligned}
& \mathbb{E}\{\Delta V(k)\} \\
&=\mathbb{E}\{[ A(p(k)) \xi(k)+D(p(k)) \\
& \times \xi(k-d)+B u(k) \\
&+N f(z(k))]^{T} Q(p(k+1)) \\
& \times {[A(p(k)) \xi(k)+D(p(k))} \\
& \times \xi(k-d)+B u(k) \\
&+N f(z(k))]+\sigma^{2} \xi^{T}(k) J^{T} M^{T} \\
& \times Q(p(k+1)) M J \xi(k) \\
&+ p(k)(1-p(k)) \\
& \times {[K(p(k)) J \xi(k)} \\
&-K(p(k)) J \xi(k-d)]^{T} \\
& \times Q(p(k+1)) \\
& \times {[K(p(k)) J \xi(k)} \\
&-K(p(k)) J \xi(k-d)] \\
&- \xi^{T}(k)\left[Q(p(k))-Q_{d}\right] \\
&\left.\times \xi(k)-\xi^{T}(k-d) Q_{d} \xi(k-d)\right\} .
\end{aligned}
$$

Besides that, by the condition of (4) and (5), (21) can be rewritten as follows:

$$
\begin{aligned}
\mathbb{E}\{\Delta V(k)\} & \\
\leq \mathbb{E}\{ & {\left[\left(A(p(k))+N F_{1} Z J\right) \xi(k)\right.} \\
& +D(p(k)) \xi(k-d)+B u(k) \\
& \left.+N f_{s}(z(k))\right]^{T} Q(p(k+1)) \\
\times & {\left[\left(A(p(k))+N F_{1} Z J\right) \xi(k)\right.}
\end{aligned}
$$

$$
\begin{aligned}
&+D(p(k)) \xi(k-d) \\
&\left.+B u(k)+N f_{s}(z(k))\right] \\
&+ p(k)(1-p(k)) \\
& \times {[K(p(k)) J \xi(k)} \\
&-K(p(k)) J \xi(k-d)]^{T} \\
& \times Q(p(k+1)) \\
& \times {[K(p(k)) J \xi(k)} \\
&-K(p(k)) J \xi(k-d)] \\
&- \xi^{T}(k)\left[Q(p(k))-Q_{d}\right] \xi(k) \\
&- \xi^{T}(k-d) Q_{d} \xi(k-d) \\
&+ \sigma^{2} \xi^{T}(k) J^{T} M^{T} \\
& \times Q(p(k+1)) M J \xi(k) \\
&\left.-2 f_{s}^{T}(z(k))\left(f_{s}(z(k))-F Z J \xi(k)\right)\right\} .
\end{aligned}
$$

From the previous analysis and $u(k) \equiv 0$, it follows that

$$
\mathbb{E}\{\Delta V(k)\} \leq \mathbb{E}\left\{\tilde{\xi}^{T}(k) \Pi \tilde{\xi}(k)\right\},
$$

where $\tilde{\xi}(k)=\left[\begin{array}{lll}\xi^{T}(k) & \xi^{T}(k-d) & f_{s}^{T}(z(k))\end{array}\right]^{T}$ and

$$
\Pi=\left[\begin{array}{ccc}
\Pi_{1} & * & * \\
\Pi_{2} & \Pi_{3} & * \\
\Pi_{4} & \Pi_{5} & \Pi_{6}
\end{array}\right],
$$

with

$$
\begin{aligned}
\Pi_{1}= & {\left[A(p(k))+N F_{1} Z J\right]^{T} } \\
& \times Q(p(k+1)) \\
& \times\left[A(p(k))+N F_{1} Z J\right] \\
& +\sigma^{2} J^{T} M^{T} Q(p(k+1)) M J \\
& +p(k)(1-p(k)) J^{T} K^{T} \\
& \times(p(k)) Q(p(k+1)) K(p(k)) J \\
& -\left(Q(p(k))-Q_{d}\right),
\end{aligned}
$$




$$
\begin{aligned}
\Pi_{2}= & D^{T}(p(k)) Q(p(k+1)) \\
& \times\left[A(p(k))+N F_{1} Z J\right] \\
- & p(k)(1-p(k)) J^{T} K^{T} \\
& \times(p(k)) Q(p(k+1)) K(p(k)) J, \\
\Pi_{3}= & D^{T}(p(k)) Q(p(k+1)) \\
& \times D(p(k))+p(k)(1-p(k)) \\
& \times J^{T} K^{T}(p(k)) Q(p(k+1)) \\
& \times K(p(k)) J-Q_{d},
\end{aligned}
$$

$$
\begin{aligned}
\Pi_{4}= & N^{T} Q(p(k+1)) \\
& \times\left[A(p(k))+N F_{1} Z J\right]+F Z J, \\
\Pi_{5}= & N^{T} Q(p(k+1)) D(p(k)), \\
\Pi_{6} & =N^{T} Q(p(k+1)) N-2 I .
\end{aligned}
$$

In the following, we will show that $\Pi<0$ from (17). From the relationship $-Q(p(k+1))+S+S^{T}>0$ in (17), we can see that $S$ is nonsingular. Performing congruence transformation $\operatorname{diag}\left\{I, I, I, \sigma^{-2} S^{-1}, \theta^{-1}(k) S^{-1}, S^{-1}\right\}$ to (17), we have

$$
\left[\begin{array}{cccccc}
Q_{d}-Q(p(k)) & * & * & * & * & * \\
0 & -Q_{d} & * & * & * & * \\
F Z J & 0 & -2 I & * & * & * \\
M J & 0 & 0 & -\sigma^{-2} \bar{\Gamma}(k) & * & * \\
K(p(k)) J & -K(p(k)) J & 0 & 0 & -\theta^{-1}(k) \bar{\Gamma}(k) & * \\
A(p(k))+N F_{1} Z J & D(p(k)) & N & 0 & 0 & -\bar{\Gamma}(k)
\end{array}\right]<0
$$

where, $\bar{\Gamma}(k)=-S^{-T} Q(p(k+1)) S^{-1}+S^{-1}+S^{-T}$.

Since $Q^{-1}(p(k+1))$ is positive definite, we have

$$
\begin{aligned}
Q^{-1} & (p(k+1))+S^{-T} Q(p(k+1)) S^{-1}-S^{-1}-S^{-T} \\
= & {\left[S^{-T}-Q^{-1}(p(k+1))\right] } \\
& \times Q(p(k+1)) S^{-1} \\
& -\left[S^{-T}-Q^{-1}(p(k+1))\right]
\end{aligned}
$$

$$
\begin{aligned}
= & {\left[S^{-1}-Q^{-1}(p(k+1))\right]^{T} } \\
& \times Q(p(k+1)) \\
& \times\left[S^{-1}-Q^{-1}(p(k+1))\right] \geq 0,
\end{aligned}
$$

therefore $-Q^{-1}(p(k+1)) \leq S^{-T} Q(p(k+1)) S^{-1}-S^{-1}-S^{-T}$. Then, it follows from inequality (27) that

$$
\left[\begin{array}{cccccc}
Q_{d}-Q(p(k)) & * & * & * & * & * \\
0 & -Q_{d} & * & * & * & * \\
F Z J & 0 & -2 I & * & * & * \\
M J & 0 & 0 & -\sigma^{-2} \Gamma(k) & * & * \\
K(p(k)) J & -K(p(k)) J & 0 & 0 & -\theta^{-1}(k) \Gamma(k) & * \\
A(p(k))+N F_{1} Z J & D(p(k)) & N & 0 & 0 & -\Gamma(k)
\end{array}\right]<0,
$$

with $\Gamma(k)=Q^{-1}(p(k+1))$. By Schur complement (Lemma 6), we can see that $\Pi<0$. Subsequently, we have

$$
\mathbb{E}\{\Delta V(k)\}<\lambda_{\min }(\Pi) \mathbb{E}|\tilde{\xi}(k)|^{2}
$$

where $\lambda_{\min }(\Pi)$ is the minimum eigenvalue of $\Pi$. Finally, we can confirm that the augmented system (11) is exponentially stable in mean square sense and the proof of this theorem is thus complete.
In Theorem 7, the sufficient condition ensuring the exponential stability of augmented filtering dynamics (11) has been obtained. Now, we will consider the $H_{\infty}$ performance for this dynamics under the zero initial condition.

Theorem 8. If there exist positive-definite matrix $Q_{d}>0$, $Q(p(k))>0$, and matrix $S$ such that the following matrix inequalities hold: 


$$
\left[\begin{array}{ccccccccc}
Q_{d}-Q(p(k)) & * & * & * & * & * & * & * & * \\
0 & -Q_{d} & * & * & * & * & * & * & * \\
F Z J & 0 & -2 I & * & * & * & * & * & * \\
0 & 0 & 0 & -\gamma^{2} & * & * & * & * & * \\
\sigma^{2} S^{T} M J & 0 & 0 & 0 & -\sigma^{2} \widetilde{\Gamma}(k) & * & * & * & * \\
\theta(k) \Phi_{1} & -\theta(k) \Phi_{1} & 0 & 0 & 0 & -\theta(k) \widetilde{\Gamma}(k) & * & * & * \\
\Phi_{2} & S^{T} D(p(k)) & S^{T} N & S^{T} B & 0 & 0 & -\widetilde{\Gamma}(k) & * & * \\
-E_{c d f}(p(k)) & -p(k) E_{d f}(p(k)) & 0 & 1 & 0 & 0 & 0 & -I & * \\
-\theta(k) E_{d f}(p(k)) & \theta(k) E_{d f}(p(k)) & 0 & 0 & 0 & 0 & 0 & 0 & -\theta(k)
\end{array}\right]<0,
$$

where $\theta(k)$ has been defined in (18) and

$$
\begin{gathered}
\widetilde{\Gamma}(k)=-Q(p(k+1))+S+S^{T}, \\
\Phi_{1}=S^{T} K(p(k)) J, \\
\Phi_{2}=S^{T}\left(A(p(k))+N F_{1} Z J\right)
\end{gathered}
$$

the dynamics of the augmented systems (11)-(12) are stochastically stable with disturbance attenuation level $\gamma$ under the zero initial condition.

Proof. In order to investigate the $H_{\infty}$ performance of the augmented systems (11)-(12), construct a functional as

$$
V(k):=\bar{\xi}^{T}(k) Q(p(k)) \bar{\xi}(k)+\sum_{s=k-d}^{k-1} \bar{\xi}^{T}(s) Q_{d} \bar{\xi}(s)
$$

Under the zero initial condition and (22), we have

$$
\begin{aligned}
J(N) & =\mathbb{E}\left\{\sum_{k=0}^{N}\left[e^{T}(k) e(k)-\gamma^{2} u^{T}(k) u(k)\right]\right\} \\
& \leq \mathbb{E}\left\{\sum_{k=0}^{N}\left[e^{T}(k) e(k)-\gamma^{2} u^{T}(k) u(k)+\Delta V_{k}\right]\right\} \\
& \leq \mathbb{E}\left\{\sum_{k=0}^{N} \bar{\xi}^{T}(k) \bar{\Pi} \bar{\xi}(k)\right\},
\end{aligned}
$$

where $\bar{\xi}(k)=\left[\begin{array}{llll}\xi^{T}(k) & \xi^{T}(k-d) & f_{s}^{T}(z(k)) & u^{T}(k)\end{array}\right]^{T}$ and

$$
\bar{\Pi}=\left[\begin{array}{cccc}
\bar{\Pi}_{1} & * & * & * \\
\bar{\Pi}_{2} & \bar{\Pi}_{3} & * & * \\
\bar{\Pi}_{4} & \bar{\Pi}_{5} & \bar{\Pi}_{6} & * \\
\bar{\Pi}_{7} & \bar{\Pi}_{8} & \bar{\Pi}_{9} & \bar{\Pi}_{10}
\end{array}\right],
$$

with

$$
\begin{aligned}
& \bar{\Pi}_{1}=\left[A(p(k))+N F_{1} Z J\right]^{T} \\
& \times Q(p(k+1)) \\
& \times\left[A(p(k))+N F_{1} Z J\right] \\
& +\sigma^{2} J^{T} M^{T} Q(p(k+1)) M J \\
& +p(k)(1-p(k)) J^{T} K^{T} \\
& \times(p(k)) Q(p(k+1)) \\
& \times K(p(k)) J-\left(Q(p(k))-Q_{d}\right) \\
& +E_{c d f}^{T}(p(k)) E_{c d f}(p(k)) \\
& +p(k)(1-p(k)) E_{d f}^{T} \\
& \times(p(k)) E_{d f}(p(k)), \\
& \bar{\Pi}_{2}=D(p(k))^{T} \\
& \times Q(p(k+1))\left[A(p(k))+N F_{1} Z J\right] \\
& -p(k)(1-p(k)) J^{T} K^{T} \\
& \times(p(k)) Q(p(k+1)) \\
& \times K(p(k)) J+p(k) E_{d f}^{T} \\
& \times(p(k)) E_{c d f}(p(k)) \\
& -p(k)(1-p(k)) E_{d f}^{T} \\
& \times(p(k)) E_{d f}(p(k)), \\
& \bar{\Pi}_{3}=D(p(k))^{T} Q(p(k+1)) \\
& \times D(p(k))+p(k)(1-p(k)) \\
& \times[K(p(k)) J]^{T} Q(p(k+1)) \\
& \times K(p(k)) J-Q_{d} \\
& +\left[p(k) E_{d f}(p(k))\right]^{T}
\end{aligned}
$$




$$
\begin{gathered}
\times p(k) E_{d f}(p(k))+p(k) \\
\times(1-p(k)) E_{d f}^{T}(p(k)) E_{d f}(p(k)), \\
\bar{\Pi}_{4}=N^{T} Q(p(k+1)) \\
\times\left[A(p(k))+N F_{1} Z J\right]+F Z J, \\
\bar{\Pi}_{5}=N^{T} Q(p(k+1)) D(p(k)), \\
\bar{\Pi}_{6}=N^{T} Q(p(k+1)) N-2 I, \\
\bar{\Pi}_{7}=B^{T} Q(p(k+1)) \\
\times\left[A(p(k))+N F_{1} Z J\right]-E_{c d f}^{T}(p(k)), \\
\bar{\Pi}_{8}=B^{T} Q(p(k+1)) D(p(k))-p(k) E_{d f}^{T}(p(k)), \\
\bar{\Pi}_{9}=B^{T} Q(p(k+1)) N, \\
\bar{\Pi}_{10}=B^{T} Q(p(k+1)) B+1-\gamma^{2} .
\end{gathered}
$$

By introducing a new slack matrix $S$, some mathematical techniques, and the similar proof line to the proof of
Theorem 7, we can see that $\bar{\Pi}<0$. Subsequently, letting $N \rightarrow \infty$, we have

$$
\|e(k)\|_{\mathbb{E}_{12}}<\gamma\|u(k)\|_{l_{2}} .
$$

From Definition 5 and the previous analysis we will safely come to the conclusion that the augmented systems (11)-(12) are stochastically stable with disturbance attenuation level $\gamma$ under the zero initial condition.

Remark 9. For the sake of the desired stability of the augmented systems (11), we propose a Lyapunov functional including the time-varying probability parameters, which reduces the conservatism of the sufficient condition in Theorems 7 and 8 . At the same time, in the sufficient condition, we introduce a slack variable $S$ to decouple the Lyapunov matrices and the filter parameters, which all contain the timevarying probability parameters. Such a technique can bypass the difficulty encountered in the filter design. In the following theorem, the filter design problem is dealt with.

Theorem 10. Consider the augmented systems (11)-(12). Assume that there exist positive-definite matrix sequences $\mathbb{Q}(p(k))>0, \mathbb{Q}_{d}>0$ matrix sequences $\mathfrak{A}_{f}, \mathfrak{B}_{f}, \mathfrak{夭}_{f}$, and $D_{f}$, nonsingular matrices $S_{11}, R_{2}$, and matrix $R_{1}$ such that the following parameter-dependent LMIs hold:

$$
\left[\begin{array}{ccccccccc}
Q_{d}-\mathscr{Q}(p(k)) & * & * & * & * & * & * & * & * \\
0 & -Q_{d} & * & * & * & * & * & * & * \\
F Z J & 0 & -2 I & * & * & * & * & * & * \\
0 & 0 & 0 & -\gamma^{2} & * & * & * & * & * \\
\Psi_{1} & 0 & 0 & 0 & -\sigma^{-2} \Psi_{6}(k) & * & * & * & * \\
\theta(k) \Psi_{2}(k) & -\theta(k) \Psi_{2}(k) & 0 & 0 & 0 & -\theta(k) \Psi_{6}(k) & * & * & * \\
\Psi_{3}(k) & p(k) \Psi_{2}(k) & \Psi_{4} & \Psi_{5} & 0 & 0 & -\Psi_{6}(k) & * & * \\
-\Psi_{7}(k) & -p(k) \Psi_{8}(k) & 0 & 1 & 0 & 0 & 0 & -I & * \\
-\theta(k) \Psi_{8}(k) & \theta(k) \Psi_{8}(k) & 0 & 0 & 0 & 0 & 0 & 0 & -\theta(k)
\end{array}\right]<0
$$

In this case, there exist nonsingular matrices $S_{21}$ and $S_{22}$ such that $R_{2}=S_{21}^{T} S_{22}^{-T} S_{21}$, and then the constant gains of the desired filter can be obtained as follows:

$$
\begin{gathered}
A_{f}=S_{21}^{-T} \mathfrak{A}_{f} S_{21}^{-1} S_{22}, \quad B_{f}=S_{21}^{-T} \mathfrak{B}_{f}, \\
C_{f}=\mathfrak{C}_{f} S_{21}^{-1} S_{22} .
\end{gathered}
$$

Then, there exists a desired gain-scheduled filter in the form of (8)-(9) such that the dynamics of the augmented systems (11)(12) are stochastically stable with disturbance attenuation level $\gamma$ under the zero initial condition.

Proof. Let nonsingular matrix variable $S$ in (30) be partitioned as $S=\left[S_{i j}\right]_{2 \times 2}$, where $S_{11}, S_{21}$, and $S_{22}$ are nonsingular matrices. Introduce matrices 


$$
\begin{gathered}
\mathscr{T}=\left[\begin{array}{cc}
I & 0 \\
0 & S_{22}^{-1} S_{21}
\end{array}\right], \quad \mathscr{Q}(p(k))=\mathscr{T}^{T} Q(p(k)) \mathscr{T}, \\
Q_{d}=\mathscr{T}^{T} Q_{d} \mathscr{T}, \\
R_{1}=S_{12} S_{22}^{-1} S_{21}, \quad R_{2}=S_{21}^{T} S_{22}^{-T} S_{21} .
\end{gathered}
$$

By performing congruence transformation $\operatorname{diag}\left\{\mathscr{T}^{-1}, \mathscr{T}^{-1}, I\right.$, $\left.1, \mathscr{T}^{-1}, \mathscr{T}^{-1}, \mathscr{T}^{-1}, 1,1\right\}$ to (37), we can see that (37) is equivalent to (30). It can now be concluded from Theorem 8 that (11) and (12) are stochastically stable with disturbance attenuation level $\gamma$ under the zero initial condition.
Because of the time-varying parameter $p(k)$, the number of LMIs in Theorem 10 is infinite, and all these LMIs bring enormous difficulties to solve. In the next work, we will focus on the challenge and present an effective method to overcome this difficulty.

Theorem 11. Consider the augmented systems (11)-(12). Assume that there exist positive positive-definite matrices $Q_{d}>$ $0, Q_{0}>0$, and $Q_{p}>0$, nonsingular matrices $S_{11}$ and $R_{2}$, and matrices $R_{1}, \mathfrak{A}_{f 0}, \mathfrak{A}_{f p}, \mathfrak{B}_{f 0}, \mathfrak{B}_{f p}, \mathfrak{⿰}_{f 0}, \mathfrak{⿰}_{f p}, D_{f 0}$, and $D_{f p}$, such that the following LMIs hold:

$$
\Omega^{i j r l}=\left[\begin{array}{ccccccccc}
Q_{d}-Q^{l} & * & * & * & * & * & * & * & * \\
0 & -Q_{d} & * & * & * & * & * & * & * \\
F Z J & 0 & -2 I & * & * & * & * & * & * \\
0 & 0 & 0 & -\gamma^{2} & * & * & * & * & * \\
\Psi_{1} & 0 & 0 & 0 & -\delta^{2} \Psi_{6}^{l} & * & * & * & * \\
\Psi_{2}^{i j r} & -\Psi_{2}^{i j r} & 0 & 0 & 0 & -\theta^{i j} \Psi_{6}^{l} & * & * & * \\
\Psi_{3}^{j r} & \Psi_{2}^{i r} & \Psi_{4} & \Psi_{5} & 0 & 0 & -\Psi_{6}^{l} & * & * \\
-\Psi_{7}^{j r} & -p_{i} \Psi_{8}^{r} & 0 & 1 & 0 & 0 & 0 & -1 & * \\
-\theta^{i j} \Psi_{8}^{r} & \theta^{i j} \Psi_{8}^{r} & 0 & 0 & 0 & 0 & 0 & 0 & -\theta^{i j}
\end{array}\right]<0,
$$

for $i, j, r, l=1,2$,

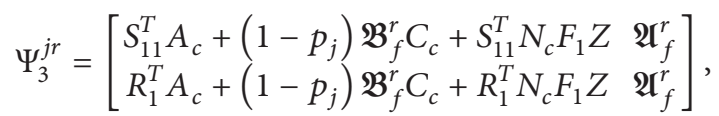

$$
\begin{aligned}
& \Psi_{2}^{i r}=p_{i}\left[\begin{array}{ll}
\mathfrak{B}_{f}^{r} C_{c} & 0 \\
\mathfrak{B}_{f}^{r} C_{c} & 0
\end{array}\right] \\
& \Psi_{6}^{l}=-\left(\widehat{Q}_{0}+p_{l} \widehat{Q}_{p}\right)+\left[\begin{array}{cc}
S_{11}+S_{11}^{T} & R_{1}+R_{2}^{T} \\
R_{2}+R_{1}^{T} & R_{2}+R_{2}^{T}
\end{array}\right], \\
& \Psi_{2}^{i j r}=p_{i}\left(1-p_{j}\right)\left[\begin{array}{ll}
\mathfrak{B}_{f}^{r} C_{c} & 0 \\
\mathfrak{B}_{f}^{r} C_{c} & 0
\end{array}\right], \\
& \Psi_{7}^{j r}=\left[\left(1-p_{j}\right) D_{f}^{r} C_{c} \quad \mathfrak{V}_{f}^{r}\right], \quad \mathfrak{U}_{f}^{r}=\mathfrak{A}_{f 0}+p_{r} \mathfrak{A}_{f p}, \\
& \mathfrak{B}_{f}^{r}=\mathfrak{B}_{f 0}+p_{r} \mathfrak{B}_{f p}, \quad \mathfrak{c}_{f}^{r}=\mathfrak{c}_{f 0}+p_{r} \mathfrak{c}_{f p}, \\
& \mathbb{Q}^{l}=Q_{0}+p_{l} Q_{p}, \quad \theta^{i j}=p_{i}\left(1-p_{j}\right), \\
& \Psi_{8}^{r}=\left[\begin{array}{ll}
D_{f}^{r} C_{c} & 0
\end{array}\right],
\end{aligned}
$$

$$
\begin{array}{cc}
A_{f 0}=S_{21}^{-T} \mathfrak{A}_{f 0} S_{21}^{-1} S_{22}, & A_{f p}=S_{21}^{-T} \mathfrak{A}_{f p} S_{21}^{-1} S_{22}, \\
B_{f 0}=S_{21}^{-T} \mathfrak{B}_{f 0}, & B_{f p}=S_{21}^{-T} \mathfrak{B}_{f p}, \\
C_{f 0}=\mathfrak{C}_{f 0} S_{21}^{-1} S_{22}, & C_{f p}=\mathfrak{S}_{f p} S_{21}^{-1} S_{22} .
\end{array}
$$

Then, a gain-scheduled filter can be obtained in the form of (8)-(9) such that the dynamics of the augmented systems (11)-

(12) are stochastically stable with disturbance attenuation level $\gamma$ under the zero initial condition.

Proof. Firstly, choose the probability-dependent Lyapunov matrices as

$$
\mathcal{Q}(p(k))=\mathbb{Q}_{0}+p(k) \mathbb{Q}_{p},
$$

where $Q_{0}>0$ and $Q_{p}>0$. Setting

$$
\lambda_{1}(k)=\frac{p_{2}-p(k)}{p_{2}-p_{1}}, \quad \lambda_{2}(k)=\frac{p(k)-p_{1}}{p_{2}-p_{1}},
$$

we have

$$
\begin{gathered}
\lambda_{1}(k)+\lambda_{2}(k)=1, \quad \lambda_{i}(k) \geq 0 \quad(i=1,2), \\
p(k)=\lambda_{1}(k) p_{1}+\lambda_{2}(k) p_{2} .
\end{gathered}
$$

Similarly, letting

$$
\mu_{1}(k)=\frac{p_{2}-p(k+1)}{p_{2}-p_{1}}, \quad \mu_{2}(k)=\frac{p(k+1)-p_{1}}{p_{2}-p_{1}},
$$

we have

$$
\begin{gathered}
\mu_{1}(k)+\mu_{2}(k)=1, \quad \mu_{l}(k) \geq 0 \quad(l=1,2), \\
p(k+1)=\mu_{1}(k) p_{1}+\mu_{2}(k) p_{2} .
\end{gathered}
$$

such that $R_{2}=S_{21}^{T} S_{22}^{-T} S_{21}$, and then the constant gains of the desired filter can be obtained as follows: 
From the previous transformations, it can be easily derived that

$$
\begin{array}{rlrl}
\mathcal{Q}(p(k)) & =\sum_{l=1}^{2} \lambda_{l}(k) \mathfrak{Q}^{l}, & \mathbb{Q}(p(k+1))=\sum_{l=1}^{2} \mu_{l}(k) \mathbb{Q}^{l}, \\
\mathfrak{A}_{f} & =\sum_{r=1}^{2} \lambda_{r}(k) \mathfrak{A}_{f}^{r}, & \mathfrak{B}_{f} & =\sum_{r=1}^{2} \lambda_{r}(k) \mathfrak{B}_{f}^{r}, \\
\mathfrak{S}_{f} & =\sum_{r=1}^{2} \lambda_{r}(k) \mathfrak{E}_{f}^{r}, & D_{f} & =\sum_{r=1}^{2} \lambda_{r}(k) D_{f}^{r} .
\end{array}
$$

And it follows from (41) that

$$
\sum_{i, j, r, l=1}^{2} \lambda_{i}(k) \lambda_{j}(k) \lambda_{r}(k) \mu_{l}(k) \Omega^{i j r l}<0 .
$$

Also, it follows from (46) and (48)-(50) that (37) holds, and the proof is now complete.

Remark 12. In Theorem 11, we convert infinite LMIs to finite ones by turning the time-varying parameter $p(k)$ into the polytopic form. By such a transformation, the constant gains of the desired gain-scheduled filter can be easily derived in terms of the available LMI toolbox by using the computationally appealing gain-scheduled deconvolution filter design algorithm listed as follows.

Algorithm 13. The gain-scheduled filter design algorithm.

Step 1. Given the initial values for the positive integer $N_{q}$, the initial state $\rho$, the constants $p_{1}$ and $p_{2}$, and the matrices $A_{c}$, $B_{c}, C_{c}, M_{c}, N_{c}, F_{1}, F_{2}$, and $Z$ select appropriate initial state estimate $\rho_{f}$, and set $k=0$.

Step 2. Solve the LMI in (41) to obtain the positive-definite matrices $\mathbb{Q}_{0}, \mathbb{Q}_{p}$, and $\mathbb{Q}_{d}$, matrices $\mathfrak{A}_{f 0}, \mathfrak{A}_{f p}, \mathfrak{B}_{f 0}, \mathfrak{B}_{f p}$, $\mathfrak{C}_{f 0}, \mathfrak{C}_{f p}, R_{1}, R_{2}$, and $S_{11}$. Choose appropriate nonsingular matrices $S_{21}$ to derive $A_{f 0}, A_{f p}, B_{f 0}, B_{f p}, C_{f 0}$, and $C_{f p}$.

Step 3. Based on the measured time-varying parameter $p(k)$, derive the filter gains $A_{f}, B_{f}, C_{f}$, and $D_{f}$ by (10), the state $x_{f}(k+1)$ by $(9)$, and the estimation of $u(k)$. Then, set $k=k+1$.

Step 4. If $k<N$, then go to Step 3; otherwise go to Step 5 .

Step 5. Stop.

Remark 14. In Algorithm 13, detailed steps have been given for the gain-scheduled deconvolution filter design problem according to Theorem 11. By employing this algorithm along with the LMI toolbox, the time-varying filter gains can be easily derived from the measured/estimated time-varying missing probability $p(k)$ in real time.

\section{An Illustrative Example}

In this section, an example is given to design the deconvolution filters for stochastic systems with randomly occurring sensor delays.
The system parameters are given as follows:

$$
\begin{aligned}
& A_{c}=\left[\begin{array}{cc}
0.601 & -0.065 \\
0 & 0.420
\end{array}\right], \quad M_{c}=\left[\begin{array}{cc}
0.013 & 0 \\
0 & 0.024
\end{array}\right] \text {, } \\
& N_{c}=\left[\begin{array}{cc}
0.014 & 0 \\
0 & 0.062
\end{array}\right], \quad Z=\left[\begin{array}{cc}
0.291 & 0 \\
0 & 0.599
\end{array}\right] \text {, } \\
& F_{1}=\left[\begin{array}{cc}
0.159 & 0 \\
0 & 0.311
\end{array}\right], \quad F_{2}=\left[\begin{array}{cc}
0.409 & 0 \\
0 & 1.501
\end{array}\right] \text {, } \\
& C_{c}=\left[\begin{array}{ll}
0.22 & 0.075
\end{array}\right], \quad B_{c}=\left[\begin{array}{l}
0.109 \\
0.081
\end{array}\right] \text {, } \\
& p_{1}=0.23, \quad p_{2}=0.45, \quad \sigma=1 .
\end{aligned}
$$

The measurable time-varying probability sequence is assumed as $0.23 e^{0.0168 k}$. Then, the constant filter parameters $A_{f 0}, A_{f p}, B_{f 0}, B_{f p}, C_{f 0}, C_{f p}, D_{f 0}$, and $D_{f p}$ can be obtained as follows:

$$
\begin{gathered}
A_{f 0}=\left[\begin{array}{cc}
0.3998 & 0.2138 \\
-0.1733 & -0.0501
\end{array}\right], \quad B_{f 0}=\left[\begin{array}{c}
-0.0521 \\
0.0364
\end{array}\right], \\
C_{f 0}=\left[\begin{array}{ll}
-0.0266 & -0.0249
\end{array}\right], \quad D_{f 0}=16, \\
A_{f p}=\left[\begin{array}{ll}
-0.0109 & -0.0072 \\
-0.0246 & -0.0160
\end{array}\right], \quad B_{f p}=\left[\begin{array}{c}
-0.0230 \\
0.0020
\end{array}\right], \\
C_{f p}=\left[\begin{array}{ll}
-0.0794 & -0.0520
\end{array}\right], \quad D_{f p}=17 .
\end{gathered}
$$

Figure 2 includes the response curves of input signal $u(k)$ and the simulation results of estimation $\widehat{u}(k)$. Figure 3 gives the time-varying missing probability $p(k)$, and the corresponding filter parameters are given in Table 1 . The simulation results have illustrated the rationality and effectiveness of the previous theoretical analysis.

\section{Conclusions}

This paper has dealt with the deconvolution filtering problem for a class of discrete-time stochastic systems with randomly occurring sensor delays, nonlinear disturbances, and external stochastic noises. We assume the sensor delays to be randomly occurring, and the occurring way is modeled by a stochastic variable sequence satisfying time-varying Bernoulli distributions. A sufficient condition has been derived to guarantee the stability of the considered stochastic systems by constructing probability-dependent Lyapunov functional and employing convex optimization method. Through some mathematical transformation, we convert the matrix inequalities into solvable form, and then a finite set of inequalities for designing the desired filter are obtained. The proposed gain-scheduled filters include both constant parameters and time-varying gains which can be updated online according to the measurable missing probabilities in real time. The desired filters can be obtained by solving a set of LMIs relying on the time-varying feature of sensor delays. By using the obtained filter, we can accurately estimate the input signal distorted by the noisy transmission channel and the delayed sensor outputs. A simulation example is exploited to illustrate the effectiveness of the proposed design scheme. 
TABLE 1: The time-varying filter parameters.

\begin{tabular}{|c|c|c|c|c|c|}
\hline$k$ & $p(k)$ & $A_{f}$ & $B_{f}$ & $C_{f}$ & $D_{f}$ \\
\hline 1 & 0.2339 & {$\left[\begin{array}{cc}0.3972 & 0.2121 \\
-0.1790 & -0.0539\end{array}\right]$} & {$\left[\begin{array}{c}-0.0574 \\
0.0369\end{array}\right]$} & {$\left[\begin{array}{ll}-0.0452 & -0.0370\end{array}\right]$} & 19.9762 \\
\hline 2 & 0.2379 & {$\left[\begin{array}{cc}0.3972 & 0.2121 \\
-0.1791 & -0.0539\end{array}\right]$} & {$\left[\begin{array}{c}-0.0575 \\
0.0369\end{array}\right]$} & {$\left[\begin{array}{ll}-0.0455 & -0.0373\end{array}\right]$} & 20.0436 \\
\hline 3 & 0.2419 & {$\left[\begin{array}{cc}0.3971 & 0.2121 \\
-0.1792 & -0.0540\end{array}\right]$} & {$\left[\begin{array}{c}-0.0576 \\
0.0369\end{array}\right]$} & {$\left[\begin{array}{ll}-0.0458 & -0.0375\end{array}\right]$} & 20.1121 \\
\hline 4 & 0.2460 & {$\left[\begin{array}{cc}0.3971 & 0.2120 \\
-0.1793 & -0.0541\end{array}\right]$} & {$\left[\begin{array}{c}-0.0577 \\
0.0369\end{array}\right]$} & {$\left[\begin{array}{ll}-0.0461 & -0.0377\end{array}\right]$} & 20.1818 \\
\hline$\vdots$ & $\vdots$ & $\vdots$ & $\vdots$ & $\vdots$ & $\vdots$ \\
\hline
\end{tabular}

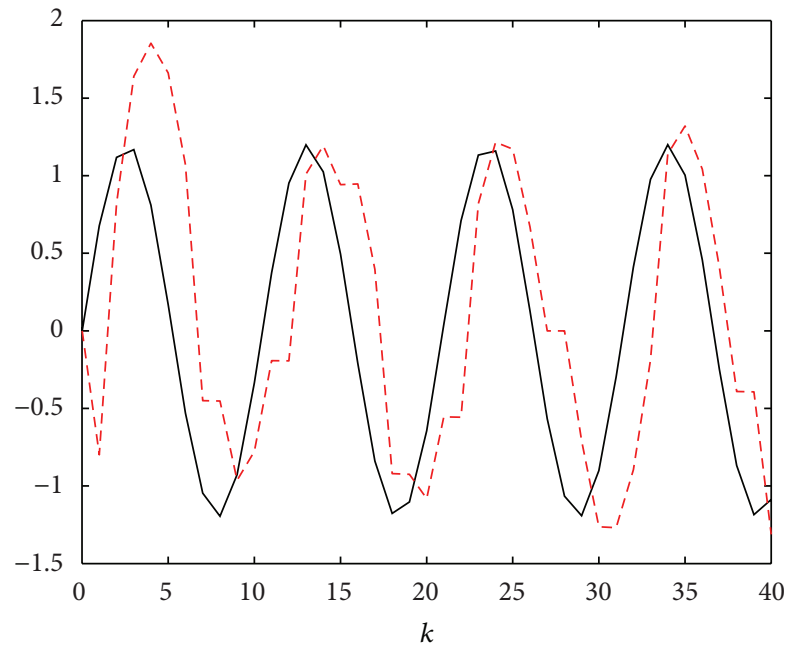

_ The trajectory of signal $u(k)$

FIGURE 2: The input signal $u(k)$ and estimation $\widehat{u}(k)$.

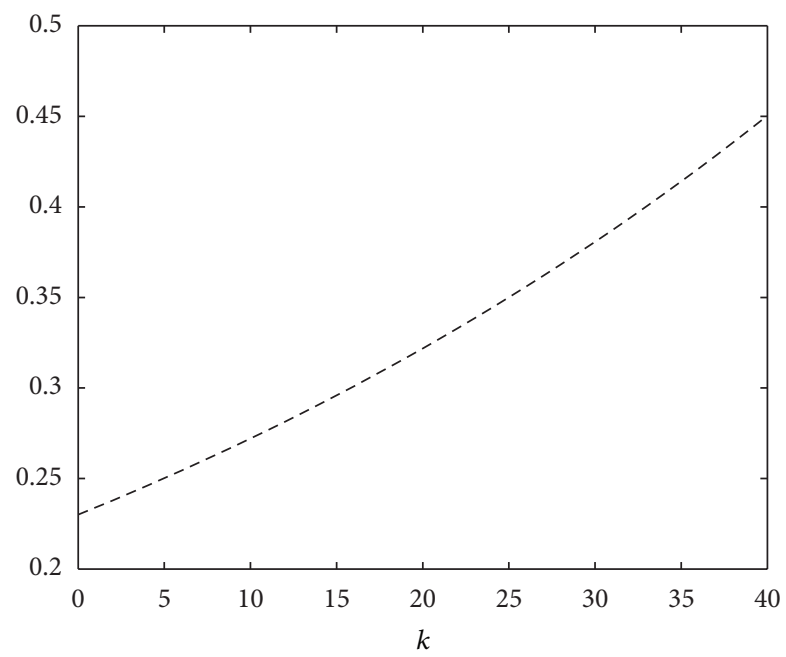

- - - The time-varying probability $p(k)$

FIGURE 3: The time-varying probability $p(k)$.

\section{Acknowledgments}

This work was supported in part by the National Natural Science Foundation of China under Grant 61074016, the Program for Professor of Special Appointment (Eastern Scholar) at Shanghai Institutions of Higher Learning, the Program for New Century Excellent Talents in University under Grant NCET-11-1051, the Leverhulme Trust of the UK, and the Alexander von Humboldt Foundation of Germany.

\section{References}

[1] A. Casavola, D. Famularo, and G. Franzè, "A robust deconvolution scheme for fault detection and isolation of uncertain linear systems: an LMI approach," Automatica, vol. 41, no. 8, pp. 1463 $1472,2005$.

[2] S. Gelfand and B. Chen, "Minimax robust deconvolution filters under stochastic parametric and noise uncertainties," IEEE Transactions on Signal Processing, vol. 42, no. 1, pp. 32-44, 1994.

[3] S. Peng and B. Chen, "A deconvolution filter for multichannel nonminimum phase systems via the minimax approach," Signal Processing, vol. 36, pp. 71-90, 1994.

[4] B. Shen, Z. Wang, H. Shu, and G. Wei, " $H_{\infty}$ filtering for nonlinear discrete-time stochastic systems with randomly varying sensor delays," Automatica, vol. 45, no. 4, pp. 1032-1037, 2009.

[5] B. Shen, Z. Wang, J. Liang, and Y. Liu, "Recent advances on filtering and control for nonlinear stochastic complex systems with incomplete information: a survey," Mathematical Problems in Engineering, vol. 2012, Article ID 530759, 16 pages, 2012.

[6] Z. Wang and D. W. C. Ho, "Robust filtering under randomly varying sensor delays with variance constraints," IEEE Transactions on Circuits and Systems, vol. 51, no. 6, pp. 320-326, 2004.

[7] D. Ding, Z. Wang, B. Shen, and H. Shu, " $H_{\infty}$ state estimation for discrete-time complex networks with randomly occurring sensor saturations and randomly varying sensor delayss," IEEE Transactions on Neural Networks and Learning Systems, vol. 23, no. 5, pp. 725-736, 2012.

[8] A. Ray, "Output feedback control under randomly varying distributed delays," Journal of Guidance Control and Dynamics, vol. 17, no. 4, pp. 701-711, 1994.

[9] F. Yang, Z. Wang, G. Feng, and X. Liu, "Robust filtering with randomly varying sensor delay: the finite-horizon case," IEEE Transactions on Circuits and Systems I, vol. 56, no. 3, pp. 664672, 2009.

[10] H. Dong, Z. Wang, and H. Gao, "Robust $H_{\infty}$ filtering for a class of nonlinear networked systems with multiple stochastic 
communication delays and packet dropouts," IEEE Transactions on Signal Processing, vol. 58, no. 4, pp. 1957-1966, 2010.

[11] X. Lu, L. Xie, H. Zhang, and W. Wang, "Robust Kalman filtering for discrete-time systems with measurement delay," IEEE Transactions on Circuits and Systems, vol. 54, no. 6, pp. 522-526, 2007.

[12] Z. Wang, Y. Liu, and X. Liu, " $H_{\infty}$ filtering for uncertain stochastic time-delay systems with sector-bounded nonlinearities," Automatica, vol. 44, no. 5, pp. 1268-1277, 2008.

[13] W.-H. Chen, Z.-H. Guan, and X. Lu, "Delay-dependent exponential stability of uncertain stochastic systems with multiple delays: an LMI approach," Systems \& Control Letters, vol. 54, no. 6, pp. 547-555, 2005.

[14] D. Ding, Z. Wang, H. Dong, and H. Shu, "Distributed $H_{\infty}$ state estimation with stochastic parameters and nonlinearities through sensor networks: the finite-horizon case," Automatica, vol. 48, no. 8, pp. 1575-1585, 2012.

[15] H. Dong, Z. Wang, and H. Gao, "Fault detection for Markovian jump systems with sensor saturations and randomly varying nonlinearities," IEEE Transactions on Circuits and Systems I, vol. 59, no. 10, pp. 2354-2362, 2012.

[16] J. Hu, Z. Wang, H. Gao, and L. K. Stergioulas, "Extended Kalman filtering with stochastic nonlinearities and multiple missing measurements," Automatica, vol. 48, no. 9, pp. 20072015, 2012.

[17] J. Hu, Z. Wang, H. Gao, and L. K. Stergioulas, "Probabilityguaranteed $H_{\infty}$ finite-horizon filtering for a class of nonlinear time-varying systems with sensor saturations," Systems \& Control Letters, vol. 61, no. 4, pp. 477-484, 2012.

[18] B. Shen, Z. Wang, H. Shu, and G. Wei, "On nonlinear $H_{\infty}$ filtering for discrete-time stochastic systems with missing measurements," IEEE Transactions on Automatic Control, vol. 53, no. 9, pp. 2170-2180, 2008.

[19] P. Apkarian and P. Gahinet, "A convex characterization of gainscheduled $H_{\infty}$ controllers," IEEE Transactions on Automatic Control, vol. 40, no. 5, pp. 853-864, 1995.

[20] C. E. de Souza and A. Trofino, "Gain-scheduled $H_{2}$ controller synthesis for linear parameter varying systems via parameterdependent Lyapunov functions," International Journal of Robust and Nonlinear Control, vol. 16, no. 5, pp. 243-257, 2006.

[21] N. T. Hoang, H. D. Tuan, P. Apkarian, and S. Hosoe, "Gainscheduled filtering for time-varying discrete systems," IEEE Transactions on Signal Processing, vol. 52, no. 9, pp. 2464-2476, 2004.

[22] W. J. Rugh and J. S. Shamma, "Research on gain scheduling," Automatica, vol. 36, no. 10, pp. 1401-1425, 2000.

[23] F. Wang and V. Balakrishnan, "Improved stability analysis and gain-scheduled controller synthesis for parameter-dependent systems," IEEE Transactions on Automatic Control, vol. 47, no. 5, pp. 720-734, 2002.

[24] S. Boyd, L. El Ghaoui, E. Feron, and V. Balakrishnan, Linear Matrix Inequalities in System and Control Theory, vol. 15, SIAM, Philadelphia, Pa, USA, 1994. 


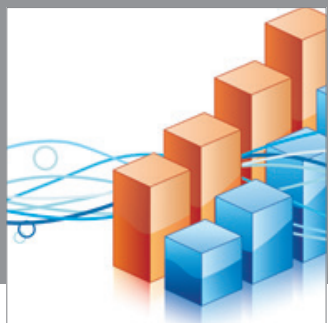

Advances in

Operations Research

mansans

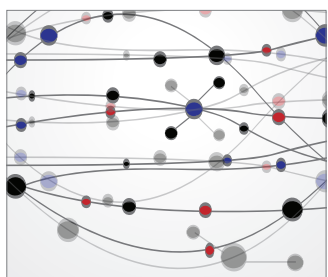

The Scientific World Journal
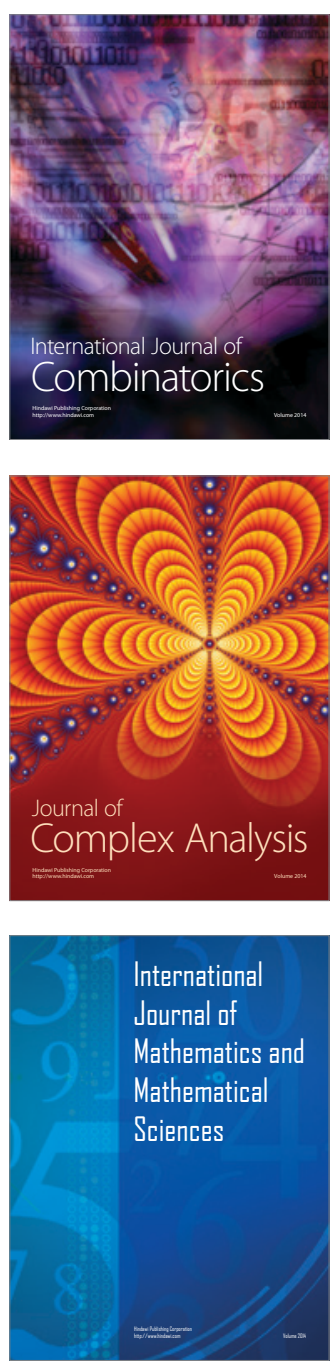
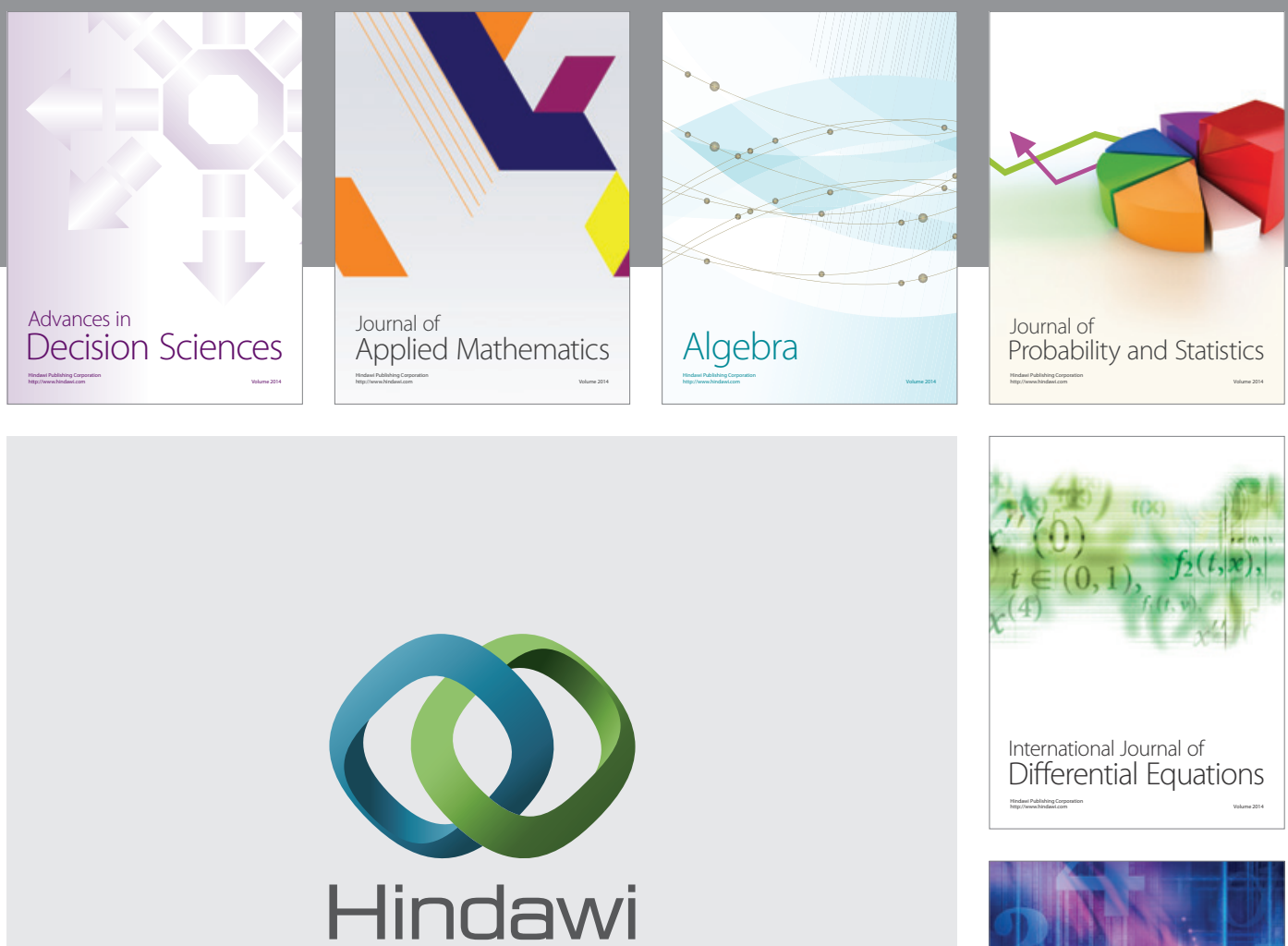

Submit your manuscripts at http://www.hindawi.com
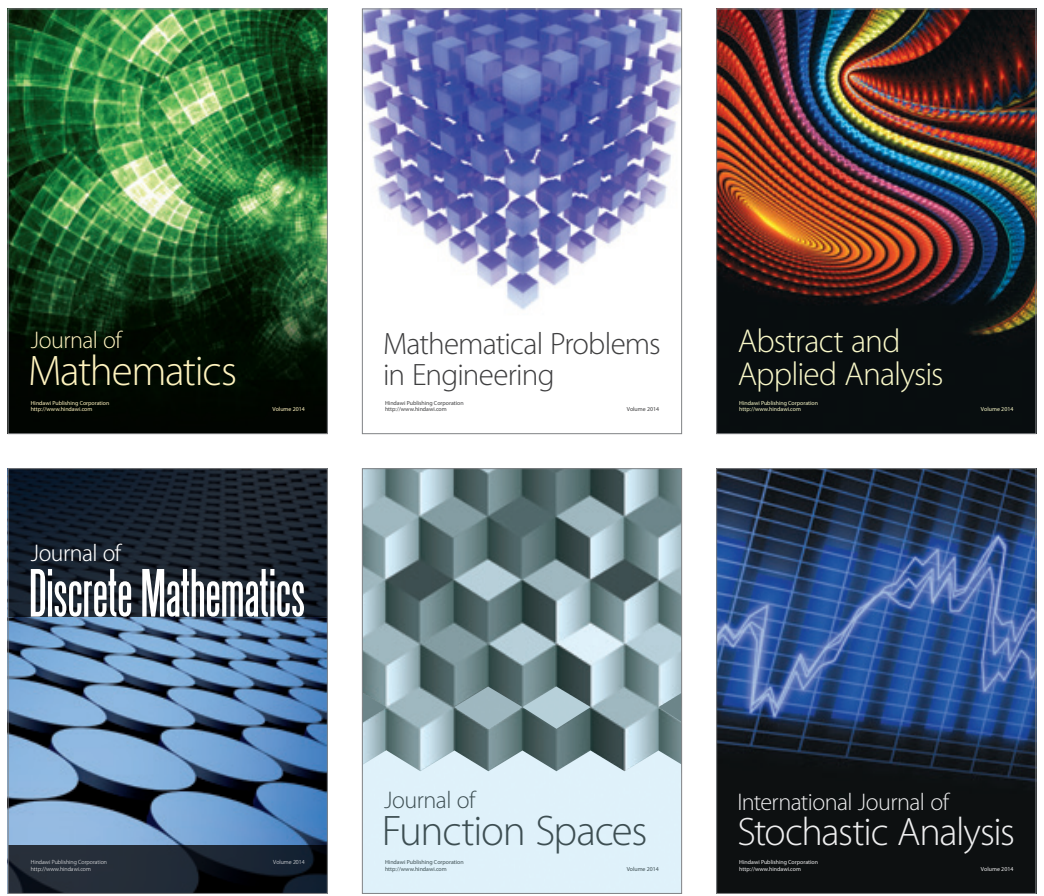

Journal of

Function Spaces

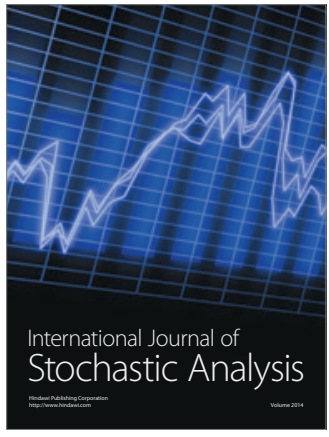

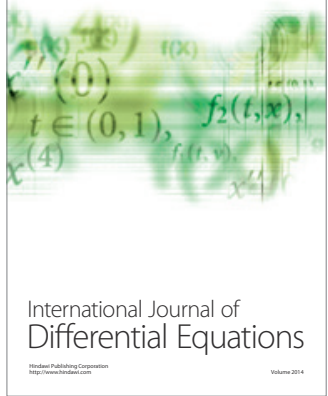
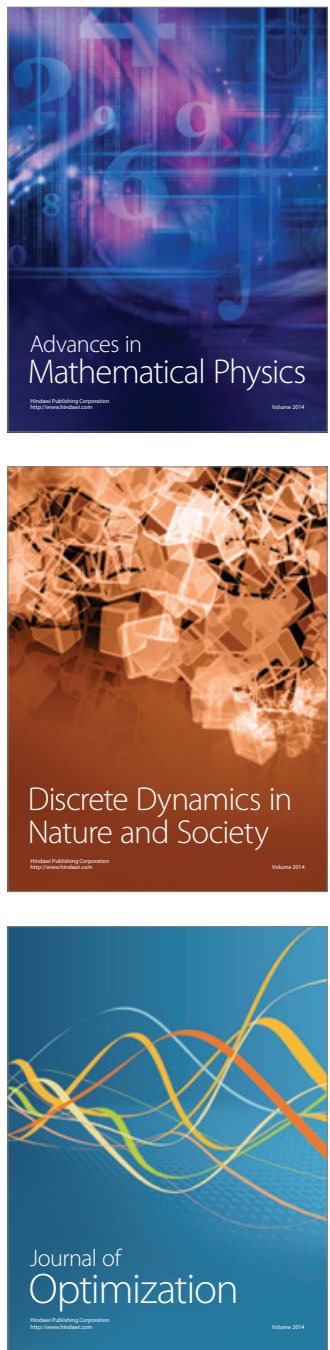